\title{
Online Appendix: \\ Social Networks as Contract Enforcement: Evidence from a Lab Experiment in the Field
}

Arun G. Chandrasekhar and Cynthia Kinnan, and Horacio Larreguy

\section{Experimental Protocol Excerpt}

The following are the experimental protocols (translated from Kannada into English) of the three games: Enforcement, No Enforcement, and No Enforcement-Savings. 


\section{Protocol}

\section{Important clarification:}

The text in italic is not meant to be read aloud to experiment participants. It has the explanation of what experimenters should do.

The remaining text that is not in italics is meant to be read aloud to experiment participants.

\section{Experiment}

Divide the research team into two groups: team $A$ and team $B$.

As participants enter the venue, team A must welcome them and locate their ID number based on their name from the individual identification list. The research team must then provide the participants with the consent forms, read the forms aloud, explain to them the contents of the forms and that the participants are free to leave at their discretion, answer any questions participants may have, and obtain their consent.

[Go to Consent Form]

Then, team A conducts the Risk Aversion and Inter-temporal Choice survey with the participants.

\section{[Go to Risk Aversion and Inter-temporal Choice Module]}

Meanwhile, based on the participants that showed up, team B uses the software on the laptop to create random pairings of ID numbers for each game in the experiment.

After completing the Risk Aversion and Inter-temporal Choice Survey, a member of team A reads the following instructions to the participants while team $B$ finishes the random pairing procedure.

\section{Experiment begins}

Thanks for coming today!

We are researchers from the Institute for Financial Management and Research (IFMR). You are participating in a study on daily decision-making. Today you will play series of short games. The information gathered here will be confidential and used for research purposes only.

\section{Overview}

We will ask you to play 3 different games today, each with several rounds. In each game you will be randomly matched with a new partner, whose identity you will find out at the beginning of each game. In each round of each game you and your partner will make some decisions. The result of these decisions will determine how much money you will earn today. 
The games will represent situations and decisions you make every day in your life. You earn some money, you save some money, you might give some money to your neighbors or friends if they are having a hard time, and you use some money to buy food, school material for your kids, clothing, etc.

\section{Explanation of payment}

Let us now discuss how you will make money today.

First, you will receive Rs for simply participating in our games.

Second, you will make money from the decisions made during the games. You will play three different types of games during today. In every round of each game you will get some income in the form of tokens. With this income you will decide how many tokens you want to consume. The experimenter will write down the amount of tokens that you want to consume on what we will call a "CONSUMPTION CHIP" and put that chip in the "CONSUMPTION BAG." Further, the experimenter will take the tokens that you wanted to consume from the ones you had.

At the end of the experiment, we will draw one "CONSUMPTION CHIP" from the "CONSUMPTION BAG” without looking. This chip will correspond to the amount of consumption that you chose to have in one round of a game. We will pay you in Rs. that amount of consumption. Importantly, this payment will solely depend on the value of the drawn chip and will be independently of the value of the other chips in your "CONSUMPTION BAG."

\section{Demonstrate.}

The "experimenter" should explain that they will be playing 3 games during the day and each game will approximately last six rounds. Then, they should expect to play approximately 18 rounds during the whole experiment. Therefore, at the end of the experiment they should expect to have put 18 "CONSUMPTION CHIPS" in the "CONSUMPTION BAG."

Then, show them a "CONSUMPTION BAG" with 18 "CONSUMPTION CHIPS" and pick one of them.

See then that the decisions you make in every round counts but you will only be paid the consumption you choose in one randomly chosen round.

Before I explain each of the three games that you will play today, are there any questions? Answer any questions that they may have.

\section{Games}

Now we will begin explaining the games. In each of the games you will be randomly assigned a partner that will be different in each game but the same in all rounds played within a game.

Pair individuals. For this have into account that individuals cannot be paired with people that they will be paired with in future treatments. 
Before I explain you the games note that the order of the following games will be randomized after I conclude their explanation. That is, I will not explain the games necessarily in the order that you will play them.

\section{Explanation of game 1: Enforcement, no saving}

The first game I will explain to you is a very simple one. In this game, you will be randomly paired with a partner, who you will talk and interact with. In every round of this game you and your partner will get some income, and "consume" and potentially share your income. However, you will not have the possibility to "save" income from one round to "consume" in a future round.

We will start the game by randomly giving you and your partner an initial income of either Rs 30 or Rs 60. In order to decide who gets the higher endowment and who gets the lower endowment, we will come to you and your partner and randomly ask one of you to draw a ball from the "ENDOWMENT BAG," which has two balls, one with a "30" and the other one with a "60." Then, one of you will take a ball without looking and will get the Rs that the ball she got says. The other one will get the other amount.

All earnings during the games will be represented by tokens, each with a value of Rs 10 . Then, for example, whoever gets the ball with "60," will be given a cup with 6 tokens that are worth Rs 60. From now on we will denote this cup the "INCOME CUP." Following the same example, contrarily, if an individual is gets the ball with "30," the individual will receive a cup with 3 tokens.

Demonstrate procedure, the objective you should have in mind is that individuals acquire a sense of the physicality of the game.

Three members of the team of experimenters should do the demonstration. Two of them should take the role of two individuals, who will be referred to as "Individual 1" and "Individual 2." The third of them should represent itself and we will refer to him/her as the "experimenter." Assume that you are the beginning of the game, the "experimenter" will go to "Individual 1 " and ask him to draw a ball from the "ENDOWMENT BAG." If the ball has a "60," the "experimenter" will give the cup with 6 tokens to "Individual 1 " and a cup with 3 tokens to "Individual 2." If the ball has a "30," the "experimenter" will do the opposite.

Now, we will explain how you get income, and can share and "consume" income in every round of the game. In each round of this game, you and your partner will receive some income. You can think about this as what you would have earned selling your crop. In each round, one of you will be lucky, and one of you will be unlucky. If you are lucky, which you can think as getting rainfall, you will receive Rs 250. If you are unlucky, which you can think as getting a drought, you will receive Rs 0 .

To decide who is lucky and receives Rs 250, and who is unlucky and receives Rs 0, we will come to you and your partner and randomly ask one of you to draw a ball from the "INCOME BAG," which has a green ball and brown ball. The green ball represents that the individual who drew a ball from the "INCOME BAG" was lucky and got an income of Rs 250 and that the other 
individual was unlucky and got no income. The brown ball represents the opposite. Then, if in one round an individual is lucky and gets a green ball, in that round the individual will be given a cup with 25 tokens. Contrarily, if an individual is unlucky and gets a brown ball, the individual will receive an empty cup.

Demonstrate procedure.

Three members of the team of experimenters should do the demonstration. Two of them should take the role of two individuals, who will be referred to as "Individual 1" and "Individual 2." The third of them should represent itself and we will refer to him/her as the "experimenter." Assume that you are in any round of the game, the "experimenter" will go to "Individual 1" and ask him to draw a ball from the "INCOME BAG." If the ball is green, the "experimenter" will give the cup with 25 tokens to "Individual 1 " and the cup with no tokens to "Individual 2." If the ball is brown, the "experimenter" will do the opposite.

We now explain how you can share income in every round. In each round, before we come to you and your partner and randomly ask one of you to draw a ball from the "INCOME BAG" to determine who is lucky and who is unlucky, you and your partner can choose if you want to share and how much you want to share of your income. This will works as a "SHARING AGREEMENT" that you have for this round. You will be able to discuss as much as you want it but, in this game, once you decide on it, we will record it, and you and you partner will be obligated to fulfill it for that round of the game. You can think of this agreement as making a decision about whether the partner who is lucky and gets Rs 250 will share some money with the partner who is unlucky and gets nothing, and how much to share. Once you decide the agreement at the beginning of the round, in this game, you cannot change it for that round of the game. However, you can decide on a different agreement at the beginning of every round.

Once you decide on a sharing agreement, we will come to you and your partner and randomly ask one of you to draw a ball from the "INCOME BAG," which will determine who is lucky and gets Rs 250 in the form of 25 tokens in an income cup, and who is unlucky and gets Rs 0 in the form of an empty income cup. We will then split the money according to the "SHARING AGREEMENT" that you have decided upon for that round. Remember that, in this game, you cannot change the "SHARING AGREEMENT" you agreed to before.

The way you will "consume" at the end of every round will be by handing the tokens you want to consume to the experimenter, who will write down the amount that you decided to "consume" on a "CONSUMPTION CHIP" and will put this chip in your "CONSUMPTION BAG." In this game, there is no possibility of savings. Then, at the end of every round, you will "consume" all the tokens that you will end up with after the implementation of the "SHARING AGREEMENT.”

After you and your partner "consume" at the end of every round, we will decide whether the game will continue on more round. We will decide this in a random way, and consequently, the length total length of each game will be also random. 
To see whether the game will continue or not at the end of each round, we will pick a ball from this box without looking. In the box, which we will call the "ENDING BOX," we have 6 balls 5 are red, and 1 is black.

Show the audience the "ENDING BOX" with 5 red balls and 1 black ball.

Once we pick a ball from the box without looking at the end of each round, if a red ball is chosen, then the game continues for another round. If the black ball is chosen, then the game has ended, and there are no more rounds of that game. Therefore, at any point when the game hasn't ended yet, there is a five out of six chance that the game will continue, since the game only ends if the black ball is chosen.

Every time the game continues to a next round, before we draw a ball from the "INCOME BAG" to see which individual is lucky and who is unlucky, you will have the chance to create a new "SHARING AGREEMENT" about how much the lucky person will share with the unlucky person, although you can use the same agreement in each round.

Now, we will demonstrate this game. But before we do that, do you have any questions? Answer any questions they may have.

Now, we will demonstrate the game.

Three members of the team of experimenters should do the demonstration. Two of them should take the role of two individuals, who will be referred to as "Individual 1" and "Individual 2." The third of them should represent himself and we will refer to him/her as the "experimenter."

So that deciding who will get an extra income in Round 1, "Individual 1" picks the ball with a " 30 " and therefore the experimenter gives "Individual 1" an initial endowment of Rs 30 in the form of 3 tokens and "Individual 2" an initial endowment of Rs 60 in the form of 6 tokens.

In the first round, "Individual 1" and "Individual 2" decide not to make any transfers to each other.

Then, the "Individual 2" will be chosen to draw a ball from the "INCOME BAG" to determine which individual is lucky and which individual is unlucky. The "Individual 2" will draw a brown ball and then "Individual 1" gets the cup with Rs 250 in the form of 25 tokens.

Then, explain that, since Individual 1 " and "Individual 2" decided not to make any transfers to each other, in the first round "Individual 1" consumes Rs 280 and "Individual 2" Rs 60. Both will give the corresponding tokens to the "experimenter," who will write down their consumption in two "CONSUMPTION CHIPS" and put these in their respective "CONSUMPTION BAGS."

Then, the "experimenter" will draw a red ball from the "ENDING BAG" and will explain that the game continues. 
Again, in the second round, "Individual 1" and "Individual 2" decide not to make any transfers to each other.

Then, "Individual 2" should draw a green ball from the "INCOME BAG" and therefore the "Individual 2" gets the cup with Rs 250 in the form of 25 tokens.

Then, explain that, since Individual 1" and "Individual 2" decided again not to make any transfers to each other, in the second round the "Individual 1" consumes Rs 0 and the "Individual 2" consumes Rs 250. Both will give the corresponding tokens to the "experimenter," who will write down their consumption in two "CONSUMPTION CHIPS" and put these in their respective "CONSUMPTION BAGS."

Then, the "experimenter" will draw a red ball from the "ENDING BAG" and will explain that the game continues.

Then, the "experimenter" should explain that the game will continue until a black ball in drawn from the "ENDING BOX."

Then, the "experimenter" should also remind that, as the probability that a game continues for another round is 5 over 6 , the participants should expect to play approximately 6 rounds during each game. The "experimenter" should explain that, for example, in this game they will assume that he drew red balls from the "ENDING BOX" in the first 7 rounds but a black one in the eighth round. Then individuals would play 8 rounds of this game. The "experimenter" should emphasize that the length of the game was random.

Then, the experimenter should explain that they will now analyze what would be the payment that "Individual 1" would get in the case that the round that is randomly chosen to decide how much money he will be paid is from this game.

Then, the "experimenter" should explain that, as this game was assume to have 8 rounds and only two were demonstrated, for the other 6 rounds, because the probability of being lucky is one half, they will assume that "Individual 1" got lucky half the times and "Individual 2" got lucky the other half of the times. Then, assuming that the individuals decided to continue not sharing any of their income, the "experimenter" will add three "CONSUMPTION CHIPS" that say Rs 250 and three "CONSUMPTION CHIPS" that say Rs 0 in "Individual 1"'s "CONSUMPTION $B A G$ " (along with the chips that are already there).

Then, the experimenter should start drawing "CONSUMPTION CHIPS" from "Individual 1"'s "CONSUMPTION BAG," where there is one chip of Rs 280 from the first round, 3 of Rs 250 from half of the last 6 rounds, and Rs 0 from the second round and half of the last 6 rounds, to make the point that it would be as likely that "Individual 1" makes Rs 250 as that he makes no money at all.

Now, we will see another demonstration so that you get a better understanding of how the game works and therefore how you can make money today. 
Three members of the team of experimenters should do the demonstration. Two of them should take the role of two individuals, who will be referred to as "Individual 1" and "Individual 2." The third of them should represent itself and we will refer to him/her as the "experimenter." So that deciding who will get a larger endowment in Round 1, "Individual 1" picks the ball with $a$ "30" and therefore the experimenter gives "Individual 1 " an initial endowment of Rs 30 in the form of 3 tokens and "Individual 2" an initial endowment of Rs 60 in the form of 6 tokens.

In the first round, "Individual 1" and "Individual 2" agree that whoever is lucky will give the other Rs. 100.

Then, the "Individual 2" will be chosen to draw a ball from the "INCOME BAG" to determine which individual is lucky and which individual is unlucky. The "Individual 2" will draw a brown ball and then "Individual 1" gets the cup with Rs 250 in the form of 25 tokens.

Then, in the first round "Individual 1 " consumes Rs 180 and "Individual 2" Rs 160. Both will give the corresponding tokens to the "experimenter," who will write down their consumption in two "CONSUMPTION CHIPS" and put these in their respective "CONSUMPTION BAGS."

Then, the "experimenter" will draw a red ball from the "ENDING BAG" and will explain that the game continues.

In the second round, "Individual 1" and "Individual 2" agree that, if "Individual 1" is lucky will give "Individual 2" Rs. 80, and that, if "Individual 2" is lucky will give "Individual 1 ” Rs. 120 .

Then, "Individual 2" should draw a green ball from the "INCOME BAG" and therefore the "Individual 2" gets the cup with Rs 250 in the form of 25 tokens.

Then, in the second round the "Individual 1" consumes Rs 120 and the "Individual 2" consumes Rs 130 .

Then, the "experimenter" will draw a red ball from the "ENDING BAG" and will explain that the game continues.

In the third round, "Individual 1" and "Individual 2" agree that whoever is lucky will give the other Rs. 100.

Then, "Individual 1 " should draw a green ball from the "INCOME BAG" and therefore the "Individual 1 " gets the cup with Rs 250 in the form of 25 tokens.

Then, in the third round the "Individual 1" consumes Rs 150 and the "Individual 2" consumes Rs 100 .

Then, the "experimenter" will draw a red ball from the "ENDING BAG" and will explain that the game continues. 
Then, the "experimenter" should remind participants that, as the probability that a game continues for another round is 5 over 6, the participants should expect to play approximately 6 rounds during each game. The "experimenter" should explain that, for example, in this game they will assume that he drew red balls from the "ENDING BOX" in the first 4 rounds but a black one in the fifth round. Then individuals would play 5 rounds of this game. The "experimenter" should emphasize that the length of the game was random.

Then, the experimenter should explain that they will now analyze what would be the payment that "Individual 2" would get in the case that the round that is randomly chosen to decide how much money he will be paid is from this game.

Then, the "experimenter" should explain that, as this game was assumed to have 5 rounds and only three were demonstrated, for the other 2 rounds, because the probability of being lucky is one half, they will assume that "Individual 1" got lucky in round 4 and "Individual 2" got lucky in round 5. That is, each individual got luck once. Further, they will assume that during these 2 rounds individuals shared 100 Rs when lucky. Then, the "experimenter" will add 1 "CONSUMPTION CHIP" that say Rs 150 and 1 "CONSUMPTION CHIP" that say Rs 100 to "Individual 2"'s "CONSUMPTION BAG" (along with the chips that are already there).

Then, the experimenter should start drawing "CONSUMPTION CHIPS" from the "Individual 2 "'s "CONSUMPTION BAG," where there is one chip with Rs 160 from the first round, another chip with Rs 130 from the second round, two chips with Rs 100 from the third and fourth round, and a last chip with 150 Rs from the fifth round, to make the point that it would be certain that "Individual 2" makes something between Rs 100 and Rs 160.

Are there any questions about the game?

Answer any questions they may have.

Now, we will practice the game. Note that this will only be practice rounds and that you will not actually play with your actual partner. You will play the actual games with your actual partners after we explain all games, practice game them and we answer any question you might have about the games.

Participants play three rounds of the game and information is recorded exactly as if the game was actually being played.

Are there any questions about the game?

Answer any questions they may have. 


\section{Explanation of game 2: No enforcement, no saving}

I will now explain a second game. Recall that the order of the following games will be chosen at random after I explain them. That is, the games are not necessarily explained in the order that you will play them.

To play this game, you will be randomly paired with a new partner, who you will talk and interact with. In every round of this game you and your partner will also get some income, and "consume" and potentially share your income. However, as in the previously explained game, you will not have the possibility to "save" income from one round to "consume" in a future round.

The only difference with respect to the previous game is that, after one of you draws a ball from the "INCOME BAG" to determine who is lucky and who is unlucky, the lucky individual can decide to share a different amount from the one established in the "SHARING AGREEMENT." That is, you will not be obligated to fulfill the "SHARING AGREEMENT," as it was the case in the previous game.

As in the previously explained game, we will start the game by randomly giving you and your partner an endowment of either Rs 30 or Rs 60. In order to decide who gets the higher endowment and who gets the lower extra endowment, we will come to you and your partner and randomly ask one of you to draw a ball from the "ENDOWMENT BAG," which you might remember has two balls, one with a "30" and the other one with a "60." Then, one of you will take a ball without looking and will get the Rs that the ball she got says. The other one will get the other amount.

The game will then continue as in the previously explained game. In each round, before we come to you and your partner and randomly ask one of you to draw a ball from the "INCOME BAG" to determine who is lucky and who is unlucky, you and your partner can decide on a "SHARING AGREEMENT" that establishes how much you and your partner would want to share of your income if lucky. You can think of this agreement as making a decision about whether the partner who is lucky and gets Rs 250 will share some money with the partner who is unlucky and gets nothing, and how much to share. Importantly, as opposed to the previously explained game, once you decide the agreement at the beginning of the round, you will be able to change your mind after it is determines who is lucky and gets Rs 250 and who is unlucky and gets Rs 0 . That is you are not obligated to fulfill the "SHARING AGREEMENT."

Then, we will come to you and your partner and randomly ask one of you draw a ball randomly from the "INCOME BAG," which has a green ball and brown ball. The ball he draws determines his income for that round. Then, if the individual that is randomly chosen to randomly draw a ball from the "INCOME BAG" draws a green ball, he is lucky and gets an income of Rs 250 and the other individual is unlucky and gets no income. The opposite holds when the drawn ball is brown. The experimenter will give the lucky individual a cup with 25 tokens and an empty cup to the unlucky individual. 
Then, you and your partner will be able to decide whether you want to split the money according to the "SHARING AGREEMENT" that you have decided upon for that round or not.

If you decide to split the money according to the "SHARING AGREEMENT," the lucky individual will give the corresponding tokens to the unlucky individual. If not, the lucky individual can decide how much he wants to transfer to the unlucky individual, which can vary from nothing to Rs 250.

Then, at the end of every round, you will "consume" all the tokens that you end up with after the implementation of any transfer that the lucky individual might want to make to the unlucky individual. You will then "consume" by handing such tokens to the experimenter, who will write down the amount that you decided to "consume" on a "CONSUMPTION CHIP" and will put this chip in your "CONSUMPTION BAG."

We will repeat this process until we select a black ball from the "ENDING BOX," which means that the game has ended.

Every time the game continues to a next round, before we draw a ball from the "INCOME BAG" to see which individual is lucky and who is unlucky, you will have the chance to create a new "SHARING AGREEMENT" about how much the lucky person wants share with the unlucky person.

Now, we will demonstrate this game. But before we do that, do you have any questions? Answer any questions they may have.

Now, we will demonstrate the game.

Three members of the team of experimenters should do the demonstration. Two of them should take the role of two individuals, who will be referred to as "Individual 1" and "Individual 2." The third of them should represent itself and we will refer to him/her as the "experimenter."

At the beginning of the game "Individual 1" is chosen to draw a ball from the "ENDOWMENT BAG." "Individual 1 " picks the ball with a "60" and therefore the experimenter gives "Individual 1" an initial endowment of Rs 60 in the form of 6 tokens and "Individual 2" an initial endowment of Rs 30 in the form of 3 tokens.

In the first round, "Individual 1" and "Individual 2" decide that, if lucky, they will transfer Rs 80 to each other.

Then, the "Individual 2" should draw a ball from the "INCOME BAG" to determine which individual is lucky and which individual is unlucky. The ball will be green and therefore "Individual 2" gets the cup with 25 tokens.

Then, "Individual 1 " and "Individual 2" decide to split the money according to the "SHARING AGREEMENT" that they have decided before. 
Then, in the first round "Individual 1" consumes Rs 140 and "Individual 2" Rs 200. Both will give the corresponding tokens to the "experimenter," who will write down their consumption in two "CONSUMPTION CHIPS" and put these in their respective "CONSUMPTION BAGS."

Then, the "experimenter" will draw a red ball from the "ENDING BAG" and will explain that the game continues.

"Individual 1" and "Individual 2" decide that, if lucky, "Individual 1" will give Rs 100 to "Individual 2" and, if lucky, "Individual 2" will give Rs 80 to "Individual 1."

Then, the "Individual 2" should draw a brown ball from the "INCOME BAG" and therefore the "Individual 1" gets the cup with Rs 250.

Then, "Individual 1" and "Individual 2" decide to split the money according to the "SHARING AGREEMENT" that they have decided before.

Then, in the second round the "Individual 1" consumes Rs 150 and the "Individual 2" consumes Rs 100. Both will give the corresponding tokens to the "experimenter," who will write down their consumption in two "CONSUMPTION CHIPS" and put these in their respective "CONSUMPTION BAGS."

Then, the "experimenter" will draw a red ball from the "ENDING BAG" and will explain that the game continues.

Then, the "experimenter" should explain that the game will continue until a black ball is drawn from the "ENDING BOX."

Then, the "experimenter" should also explain that, as the probability that a game continues for another round is 5 over 6 , the participants should expect to play approximately 6 rounds during each game. The "experimenter" should explain that, for example, in this game they will assume that he drew red balls from the "ENDING BOX" in the first 6 rounds but a black one in the seventh round. Then individuals would play 7 rounds of this game. The "experimenter" should emphasize that the length of the game was random.

The experimenter should then explain that they will now analyze what would be the payment that "Individual 1" would get in the case that the round that is randomly chosen to decide how much money he will be paid is from this game.

Then, the "experimenter" should explain that, as this game was assume to have 7 rounds and only two were demonstrated, for the other 5 rounds, because the probability of being lucky is one half, they will assume that "Individual 1" got lucky 2 of the 5 times and "Individual 2" got lucky 3 of the 5 of the times. Further, they will assume that in every round "Individual 1 " and "Individual 2" agree to transfer each other Rs 100 when lucky and after seeing who is lucky and who is unlucky they decide to split their income according to their "SHARING AGREEMENT." Then, the "experimenter" will add two "CONSUMPTION CHIPS" that say Rs 150 and three 
"CONSUMPTION CHIPS" that say Rs 100 in "Individual 1"'s "CONSUMPTION BAG" along with the chips that are already there (one of Rs 140 and one of Rs 150).

Then, the experimenter should start drawing "CONSUMPTION CHIPS" from the "Individual 1 "'s "CONSUMPTION BAG," where there is one chip of Rs 140 from round one, 3 chips with Rs 150 from the second round and the 2 rounds that the "Individual 1" is lucky in the last 5 rounds, and 3 chips with Rs 100 from the 3 rounds that the "Individual 1 " is unlucky in the last 5 round, to make the point that it is certain that "Individual 1" will make between Rs 100 and Rs 150 .

Now, we will see another demonstration so that you get a better understanding of how the game works and therefore how you can make money today.

Three members of the team of experimenters should do the demonstration. Two of them should take the role of two individuals, who will be referred to as "Individual 1" and "Individual 2." The third of them should represent itself and we will refer to him/her as the "experimenter."

At the beginning of the game "Individual 2" picks the ball with a "60" and therefore the experimenter gives "Individual 1" an initial endowment of Rs 30 in the form of 3 tokens and "Individual 2" an initial endowment of Rs 60 in the form of 6 tokens.

In the first round, "Individual 1" and "Individual 2" agree that whoever is lucky will give the other Rs. 80.

Then, the "Individual 2" should draw a ball from the "INCOME BAG" to determine which individual is lucky and which individual is unlucky. The ball will be brown and therefore "Individual 2" gets the cup with Rs 250.

Then, "Individual 1" and "Individual 2" decide to split the money according to the "SHARING AGREEMENT" that they have decided before.

Then, in the first round "Individual 1" consumes Rs 200 and "Individual 2" Rs 140. Both will give the corresponding tokens to the "experimenter," who will write down their consumption in two "CONSUMPTION CHIPS" and put these in their respective "CONSUMPTION BAGS."

Then, the "experimenter" will draw a red ball from the "ENDING BAG" and will explain that the game continues.

In the second round, "Individual 1" and "Individual 2" agree that, if "Individual 1" is lucky will give "Individual 2" Rs. 80, and that, if "Individual 2" is lucky will give "Individual 1" Rs. 120 .

Then, the "Individual 2" should draw a green ball from the "INCOME BAG" and therefore "Individual 2" gets the cup with Rs 250 in the form of 25 tokens. 
Then, "Individual 2" decides not to split the money according to the "SHARING AGREEMENT" that they have decided before, and decides to keep the Rs 250, in the form of 25 tokens, for himself.

Then, in the second round, the "Individual 1" consumes Rs 0 and the "Individual 2" consumes Rs 250 .

Then, the "experimenter" will draw a red ball from the "ENDING BAG" and will explain that the game continues.

In the third round, "Individual 1" and "Individual 2" will choose not to share anything with each other.

Then, the "Individual 1" should draw a green ball from the "INCOME BAG" and therefore "Individual 1" gets the cup with Rs 250 in the form of 25 tokens.

Then, in the third round the "Individual 1" consumes Rs 250 and the "Individual 2" consumes Rs 0 .

Then, the "experimenter" will draw a red ball from the "ENDING BAG" and will explain that the game continues.

In the fourth round, "Individual 1" and "Individual 2" will again choose not to share anything.

Then, the "Individual 1" should draw a brown ball from the "INCOME BAG" and therefore "Individual 2" gets the cup with Rs 250 in the form of 25 tokens.

Then, in the fourth round the "Individual 1" consumes Rs 0 and the "Individual 2" consumes Rs 250.

Then, the "experimenter" will draw a red ball from the "ENDING BAG" and will explain that the game continues.

In the fifth round, "Individual 1" and "Individual 2" decide to start sharing again. Then, they agree that whoever is lucky will give the other Rs. 60.

Then, the "Individual 2" should draw a brown ball from the "INCOME BAG" and therefore "Individual 1" gets the cup with Rs 250 in the form of 25 tokens.

Then, "Individual 1" and "Individual 2" decide to split the money according to the "SHARING AGREEMENT" that they have decided before.

Then, in the fifth round the "Individual 1" consumes Rs 190 and the "Individual 2" consumes Rs 60 . 
Then, the "experimenter" will draw a black ball from the "ENDING BAG" and will explain that the game ends.

Then, the experimenter should explain that they will now analyze what would be the payment that "Individual 1" would get in the case that the round that is randomly chosen to decide how much money he will be paid is from this game.

The experimenter should start drawing "CONSUMPTION CHIPS" from the "Individual 1"'s "CONSUMPTION BAG," where there is one chip with Rs 200 from the first round, two chips with Rs 0 from the second and fourth rounds, another chip with Rs 250 from the third round, and one last chip with Rs 190 from the fifth round, to make the point that "Individual 1" should expect to consume between Rs 190 and Rs 200 for the rounds they choose to make a "SHARING AGREEMENT" where they share income and did not change their mind but between Rs 250 and Rs 0 for the rounds they choose not to make a "SHARING AGREEMENT" or they choose to not split the money according to the ones where they proposed to share income.

Are there any questions about the game?

Answer any questions they may have.

Now, we will practice the game. Note that this will only be practice rounds and that you will not actually play with your actual partner. You will play the actual games with your actual partners after we explain all games, practice game them and we answer any question you might have about the games.

Participants play three rounds of the game and information is recorded exactly as if the game was actually being played.

Are there any questions about the game?

Answer any questions they may have.

\section{Explanation of game 3: No enforcement, saving}

I will now explain you the third and last game. Recall that the order of the following games will be randomly chosen after I conclude the explanations. That is, the games are not necessarily explained in the order that you will play them.

To play this game, you will be randomly paired with a new partner, who you will talk and interact with. In every round of this game you and your partner will get also some income, and "consume" and potentially share your income.

The only difference with respect to the previous game is that you and your partner will not have to consume all your money after every round. Instead, each will be able to "save" money for the next round, or use savings you already have to have more consumption than the income you end up with at the end of a particular round. 
As in the previously explained game, we will start the game by randomly giving you and your partner an endowment of either Rs 30 or Rs 60. In order to decide who gets the higher endowment and who gets the lower extra endowment, we will come to you and your partner and randomly ask one of you to draw a ball from the "ENDOWMENT BAG," which you might remember has two balls, one with a " 30 " and the other one with a "60." Then, one of you will take a ball without looking and will get the Rs that the ball she got says. The other one will get the other amount.

The game will then continue as in the previously explained game. In each round, before we come to you and your partner and randomly ask one of you to draw a ball from the "INCOME BAG" to determine who is lucky and who is unlucky, you and your partner can decide on a "SHARING AGREEMENT" that establishes how much you and your partner would want to share of your income if lucky. Importantly, as in the previously explained game but contrary to the first game I explained to you, once you decide the agreement at the beginning of the round, you will be able to change your mind after a ball is drawn from the "INCOME BAG" to determine who is lucky and who is unlucky. That is, you can make any "SHARING AGREEMENT," but you are not obligated to follow that agreement.

Then, we will come to you and your partner and randomly ask one of you draw a ball randomly from the "INCOME BAG," which has a green ball and brown ball. The ball he draws determines his income for that round. Then, if the individual that is randomly chosen to draw a ball from the "INCOME BAG" draws a green ball he is lucky and gets an income of Rs 250 and the other individual is unlucky and gets no income. The opposite holds when the drawn ball is brown. The experimenter will instead give the individual that draws the ball an empty cup and a cup with 25 tokens to her partner.

Then, you and your partner will be able to decide whether you want to split the money according to the "SHARING AGREEMENT" that you have decided upon for that round or not. If you decide to split the money according to the "SHARING AGREEMENT," the lucky individual will give the corresponding tokens to the unlucky individual. If not, the lucky individual can decide how much he wants to transfer to the unlucky individual, which can vary from nothing to Rs 250.

While in the previous two games all the money that individuals had at the end of each round had to be consumed, this is no longer the case in this game. After deciding any transfers that the lucky individual might want to make to the unlucky one, following or not the "SHARING AGREEMENT," you each will choose how to split the money you end up with between what you "consume" and what you "save" for the next round. Remember that to consume you have to give the experimenter the tokens you want to consume. This one will write down your "consumption" for this round on a "CONSUMPTION CHIP" and put it in your "CONSUMPTION BAG." Further, in order to save for the future you will put the tokens that you do not want to consume in your "SAVINGS CUP." Note that this "SAVINGS CUP” belongs privately to you only.

We will repeat this process until we select a black ball from the "ENDING BOX," which means that the game has ended. Every time the game continues to a next round, before we draw a ball 
from the "INCOME BAG" to see which individual is lucky and who is unlucky, you will have the chance to create a new "SHARING AGREEMENT" about how much the lucky person wants share with the unlucky person. Also, any savings you have when a black ball is drawn, if any, will not be available for you to consume in future games.

Now, we will demonstrate this game. But before we do that, do you have any questions? Answer any questions they may have.

Now, we will demonstrate the game.

Three members of the team of experimenters should do the demonstration. Two of them should take the role of two individuals, who will be referred to as "Individual 1" and "Individual 2." The third of them should represent itself and we will refer to him/her as the "experimenter."

At the beginning of the game "Individual 1" picks the ball with a "60" and therefore the experimenter gives "Individual 1" an initial endowment of Rs 60 and "Individual 2" an initial endowment of Rs 30 .

"Individual 1" and "Individual 2" agree that whoever is lucky will give the other Rs 90.

Then, the "experimenter" should come to "Individual 2" and have him draw a ball from the "INCOME BAG" to determine which individual is lucky and which individual is unlucky. The brown will be green and therefore "Individual 2" gets the cup with Rs 250.

Then, "Individual 2" will choose to follow the agreement and transfer Rs 90 to "Individual 1."

"Individual 1" then consumes Rs 110 and "Individual 2" consumes Rs 130. Both will give the corresponding tokens to the "experimenter," who will write down their consumption in two "CONSUMPTION CHIPS" and put these in their respective "CONSUMPTION BAGS." "Individual 2" will have Rs 60 in her "SAVINGS CUP" and "Individual 1" will have Rs 40 in her "SAVINGS CUP."

Then, the "experimenter" will draw a red ball from the "ENDING BAG" and will explain that the game continues.

"Individual 1" and "Individual 2" agree that, if "Individual 1" is lucky, he will give "Individual 2 " Rs 120, and that, if "Individual 2" is lucky, he will give "Individual 1" Rs 80.

Then, the "experimenter" should come to "Individual 1" and have him draw a ball from the "INCOME BAG" to determine which individual is lucky and which individual is unlucky. The ball will be green and therefore "Individual 1" gets the cup with Rs 250. Now, "Individual 1" decides not to follow the agreement, and instead transfers nothing to "Individual 2."

Then, the "Individual 1" will choose to consume Rs 200 and "Individual 2" will choose to consume Rs 60. Both will give the corresponding tokens to the "experimenter," who will write down their consumption in two "CONSUMPTION CHIPS" and put these in their respective 
“CONSUMPTION BAGS." The “Individual 2" will have in her "SAVINGS CUP” Rs 0 and “Individual 1" Rs 90.

Then, the "experimenter" will draw a red ball from the "ENDING BAG" and will explain that the game continues.

"Individual 2" says that he does not want to transfer anything to "Individual 1 " this round. "Individual 1 " says he will not transfer anything, either.

Then, the "experimenter" should come to "Individual 1 " and have him draw a ball from the "INCOME BAG" to determine which individual is lucky and which individual is unlucky. The ball will be brown and therefore "Individual 2" gets the cup with Rs 250. No transfers are made.

Then, "Individual 1" will choose to consume Rs 90 and the "Individual 2" will choose to consume Rs 170. Both will give the corresponding tokens to the "experimenter," who will write down their consumption in two "CONSUMPTION CHIPS" and put these in their respective "CONSUMPTION BAGS." Then, "Individual 1" will have in her "SAVINGS CUP" Rs 0 and “Individual 2" Rs 80.

Then, the "experimenter" will draw a red ball from the "ENDING BAG" and will explain that the game continues.

"Individual 2" says that he does not want to transfer anything to "Individual 1" this round. "Individual 1 " says he will not transfer anything, either.

Then, the "experimenter" should come to "Individual 1 " and have him draw a ball from the "INCOME BAG" to determine which individual is lucky and which individual is unlucky. The ball will be green and therefore "Individual 1" gets the cup with Rs 250. No transfers are made.

Then, "Individual 1" will choose to consume Rs 200 and the "Individual 2" will choose to consume Rs 80. Both will give the corresponding tokens to the "experimenter," who will write down their consumption in two "CONSUMPTION CHIPS" and put these in their respective "CONSUMPTION BAGS." Then, "Individual 1" will have in her "SAVINGS CUP" Rs 50 and “Individual 2" Rs 0.

Then, the "experimenter" will draw a black ball from the "ENDING BAG" and will explain that the game ends.

The "experimenter" should also explain that, as the probability that a game continues for another round is 5 over 6, the participants should expect to play approximately 6 rounds during each game. The "experimenter" should emphasize that the length of the game was random.

Then, the experimenter should explain that they will now analyze what would be the payment that "Individual 1 " would get in the case that the randomly chosen round for which he will be paid is from this game. 
Then, the experimenter should start drawing "CONSUMPTION CHIPS" from "Individual 2" 's "CONSUMPTION BAG," where there is one chip with Rs 130 from round 1, another with Rs 60 from round 2, another with Rs 170 from round 3, and one last chip with Rs 80 from round 4, to make the point that "Individual 1" will certainly make between Rs 60 and Rs 170.

Now, we will see another demonstration so that you get a better understanding of how the game works and therefore how you can make money today.

Three members of the team of experimenters should do the demonstration. Two of them should take the role of two individuals, who will be referred to as "Individual 1" and "Individual 2." The third of them should represent itself and we will refer to him/her as the "experimenter."

At the beginning of the game "Individual 1" picks the ball with a "30" and therefore the experimenter gives "Individual 1" an initial endowment of Rs 30 in the form of 3 tokens and "Individual 2" an initial endowment of Rs 60 in the form of 6 tokens.

"Individual 1" and "Individual 2" agree that whoever is lucky will give the other Rs. 120.

Then, the "experimenter" should come to "Individual 1" and have him draw a ball from the "INCOME BAG" to determine which individual is lucky and which individual is unlucky. The ball will be green and therefore "Individual 1" gets the cup with Rs 250 in the form of 25 tokens.

Then, the "Individual 1" will choose to follow the agreement and transfer Rs 120 to "Individual 2." "Individual 1" consumes Rs 120 and "Individual 2" Rs 120. Both will give the corresponding tokens to the "experimenter," who will write down their consumption in two "CONSUMPTION CHIPS" and put these in their respective "CONSUMPTION BAGS." The "Individual 1" will have in her "SAVINGS CUP" Rs 40 and the "Individual 2" Rs 40.

Then, the "experimenter" will draw a red ball from the "ENDING BAG" and will explain that the game continues.

"Individual 1" and "Individual 2" agree that if "Individual 1" is lucky, he will give "Individual 2 " Rs. 80, and if "Individual 2" is lucky, he will give "Individual 1" Rs 100.

Then, the "experimenter" should come to "Individual 2" and have him draw a ball from the "INCOME BAG" to determine which individual is lucky and which individual is unlucky. The ball will be brown and therefore "Individual 1" gets the cup with Rs 250.

Then, the "Individual 1" will choose to follow the agreement and transfer Rs 80 to "Individual 2." "Individual 1" consumes Rs 160 and "Individual 2" Rs 120. Both will give the corresponding tokens to the "experimenter," who will write down their consumption in two "CONSUMPTION CHIPS" and put these in their respective "CONSUMPTION BAGS." The "Individual 2" will have in her "SAVINGS CUP" Rs 0 and the "Individual 1" will have Rs 50. Then, the "experimenter" will draw a red ball from the "ENDING BAG" and will explain that the game continues. 
Then, the "experimenter" should explain that the game will continue like they have been seeing but that they will stop the demonstration so that they can practice. Then, the experimenter should explain that they will now analyze what would be the payment that "Individual 2" would get in the case that the randomly chosen round for which he will be paid is from this game.

The "experimenter" should explain that this game will be assumed to have 8 rounds. Only two were demonstrated, and for the other rounds, because the probability of being lucky is one half, they will assume that "Individual 1" got lucky half the times and "Individual 2" got lucky the other half of the times. Assume that, by using savings and transfers, "Individual 2" consumed Rs 140 when he was lucky and Rs 110 when he was unlucky. Then, the "experimenter" will add three "CONSUMPTION CHIPS" that say Rs 140 and three "CONSUMPTION CHIPS" that say Rs 110 to Individual 2's "CONSUMPTION BAG" (along with the two chips with 120 that are already there).

Then, the experimenter should start drawing "CONSUMPTION CHIPS" from "Individual 2"'s "CONSUMPTION BAG" to make the point "Individual 2" is certain to make an amount between Rs 120 and Rs 160.

Are there any questions about the game?

Answer any questions they may have.

Now, we will practice the game. Note that this will only be practice rounds and that you will not actually play with your actual partner. You will play the actual games with your actual partners once you are done practicing this game and we answer any question you might have.

Participants play three rounds of the game and information is recorded exactly as if the game was actually being played.

Are there any questions about the game?

Answer any questions they may have.

\section{Games}

Now we will begin playing the games. Remember that for this we will pair you with a new partner in each of them but that you will play all rounds of every game with the same partner.

Play all games according to the order determined by the randomization code.

Now I will read aloud the partners that you have randomly been assigned to play this game.

Read out loud pairing of individuals in each game according to the randomization code. Explain that individuals cannot be paired with people that they will be paired with in future games.

Participants break into pairs and play them games. Team of experimenters record all endowment and income realizations, as well participants' decisions regarding transfers, consumption and savings, if applicable. 
Participants perform each game until a black ball in drawn from the "ENDING BOX."

\section{Payment}

Today each of you have played 3 games. Game 1 had [mention number of game rounds] rounds, game 2 had [mention number of game rounds] rounds and game 3 had [mention number of game rounds] rounds. Each of you then have [mention total number of experiment rounds] chips in your "CONSUMPTION BAG."

Before we draw a chip from your "CONSUMPTION BAG," we will make sure the number of chips you have in there is correct. If you have a different number, we will review all the information on your consumption decisions, and adjust the number and content of the chips in your "CONSUMPTION BAG" accordingly. After that we will draw randomly a chip from your "CONSUMPTION BAG" and pay you its content together with Rs 20 for having participated in our games.

Since the payment to each of you will be private we kindly ask you to leave the room and line up according to your ID number. We will call you one at the time.

Each participant enters the payment room alone. Confirm their ID number. Give them Rs. 20 for their participation. Then, randomly draw one chip in front of the participant. Show it to the participant. Pay him or her the amount shown on the chip, and have him or her sign a receipt (with an " $X$ " if they cannot write) showing the total amount paid: the amount on the drawn chip plus Rs. 20.

One the participant has left, call the next ID number. Continue until everyone has been paid.

Thanks again for coming today. Now you are free to go.

Before you leave, are there any questions?

Answer any questions that the participant may have. 